\title{
Correlações entre Comportamentos e Competências: a busca de uma universidade verde
}

\section{Correlations between Behaviors and Competencies: a search for a green university}

Valéria Garlet ${ }^{* 1}$, Rodrigo Reis Favarin ${ }^{1}$, Thiago Antonio Beuron ${ }^{1}$, Lucia Rejane da Rosa Gama Madruga ${ }^{1}$, Camilla Castilhos de Freitas Terra ${ }^{1}$, Gustavo da Rosa Borges ${ }^{1}$

${ }^{1}$ Universidade Federal de Santa Maria (UFSM), Rio Grande do Sul, Brasil.

\begin{tabular}{l}
\hline I N F O A R T I G O \\
\hline Palavras-chave: \\
Comportamentos, \\
Competências, \\
Universidade Verde.
\end{tabular}

ARTICLE INFO

\section{Keywords:}

Behaviors,

Skills,

Green University.

\begin{abstract}
RES U M O
A preocupação com o desenvolvimento sustentável se traduz em repensar as universidades de forma a torná-las modelo de sustentabilidade. Tratar do tema sustentabilidade significa tratar das atitudes e ações das pessoas. As pessoas (indivíduos) são agentes de sustentabilidade; estudar os comportamentos e competências para sustentabilidade torna-se importante para identificar quais fatores promovem a sustentabilidade e quais precisam ser trabalhados. Com base nisso, este trabalho teve como objetivo apresentar as correlações entre comportamentos e competências para a sustentabilidade e identificar as diferenças relacionadas com fatores como: no gênero, na faixa etária, no estado civil, na escolaridade, na renda, na categoria, no tempo de instituição e no exercício de cargo de chefia. A pesquisa revelase quantitativa, com base em uma amostragem não-probabilística por conveniência dos servidores públicos federais. Os achados mostram uma correlação positiva entre os dois constructos, sendo que os respondentes apresentam maiores índices de comportamento do que de competência, e diferenças entre gênero, faixa etária, escolaridade, renda e tempo de instituição. Comportamentos e competências para a sustentabilidade são fundamentais na construção de uma Universidade Verde. A partir disso, é preciso discutir sobre formas viáveis de incentivo à sustentabilidade em ambientes de educação para que, dessa forma, a sustentabilidade seja alçada a toda sociedade.
\end{abstract}

A B S T R A C T
The concern with sustainable development translates into rethinking the universities
in order to make them models of sustainability. Addressing the theme of sustainability
means dealing with people's attitudes and actions. People (individuals) are agents of
sustainability; studying behaviors and skills for sustainability becomes important in
identifying which factors promote sustainability and which need to be addressed.
Based on this, the objective of this study was to present the correlations between
behaviors and skills for sustainability and to identify the differences related to factors
such as: gender, age, marital status, schooling, income, category, duration of
employment in the institution and in the exercise of managerial position. The research
is quantitative, based on a non-probabilistic sampling for the convenience of federal
public servants of the institution. The findings show a positive correlation between the
two constructs, with respondents presenting higher behavioral indexes than
competence, and differences between gender, age group, schooling, income, and
institution time. Behaviors and competencies for sustainability are fundamental in the
construction of a Green University. From this, it is necessary to discuss feasible ways
of encouraging sustainability in educational environments so that, in this way,
sustainability is raised to every society.

* Principal contato para correspondência.

valeriagarlet@yahoo.com.br (Garlet, V.), rodrigo.favarin@hotmail.com (Favarin, R. R.), tbeuron@gmail.com (Beuron, T. A.), luciagm@ufsm.br (Madruga, L. R. R. G.), diretoria@grupocamillaterra.com.br (Terra, C. C. F.), gustavodarosaborges@gmail.com (Borges, G. R).

DOI: $\underline{\text { dx.doi.org/10.21714/1679-18272019v17Ed.p73-87 }}$

1679-1827 (C) 2019 Gest@o.org. 


\section{Introdução}

A preocupação com o desenvolvimento sustentável da sociedade promove um cuidado especial com o ambiente físico e natural e, também, com as oportunidades sociais e econômicas das pessoas, especialmente as das gerações futuras. Só a educação é capaz de modificar o comportamento das pessoas, assim, devendo ser prioridade tanto para o governo quanto para qualquer cidadão. A sustentabilidade pode ser entendida por meio do equilíbrio entre o tripé econômico (lucro), social (pessoas) e ambiental (planeta) (MELETI; FADEL; SMITH, 2012). É na busca de um equilíbrio entre essas forças que qualquer empresa deve orientar suas práticas de sustentabilidade e responsabilidade.

"O desenvolvimento sustentável é um processo evolutivo que vislumbra o crescimento da economia, a melhoria da qualidade do ambiente e da sociedade para beneficio das gerações presentes e futuras" (SEBASTIÃO, 2010, p. 20). A proposta do desenvolvimento sustentável é alinhar o crescimento econômico e comprometimento com os recursos naturais, utilizando-os da forma mais consciente no presente para não prejudicar a qualidade de vida das gerações futuras (CMMAD, 1991).

Silva et al. (2014) indicam que o uso indevido do termo sustentabilidade faz com que as diferentes adoções se afastem do conceito inicial relacionado ao desenvolvimento sustentável. Com isso, os autores desenvolveram um estudo com o objetivo de discutir os diferentes conceitos que são utilizados por oito organizações no que se refere à sustentabilidade e seus significados. Os autores observaram

que a sustentabilidade, originalmente associada a uma maior integração entre a humanidade e natureza e à manutenção de um equilíbrio dinâmico que permitisse a existência do maior número de espécies possivel, pode ser entendida de diferentes maneiras nas organizações (SILVA et al., 2014, p. 11).

Com relação às preocupações com o meio ambiente, a maior parte das empresas considera a redução do uso de recursos naturais ou do impacto ambiental; com relação às preocupações sociais atribuem-se formas de investimentos, programas e ações sociais; no geral, as organizações estudadas reduziram o conceito de sustentabilidade buscando o atendimento das necessidades da própria organização.

As Instituições de Ensino Superior (IES), por sua vez, dispõem de um considerável compromisso quando o assunto é a formação dos cidadãos e a responsabilidade - tanto destes cidadãos quanto da própria instituição perante a sociedade. Gazzoni et al. (2018, p. 49) propõem que as IES "possuem papel fundamental na formação de pensamentos e opiniões, sendo um dos principais órgãos que podem potencializar o desenvolvimento de um pensamento sustentável". Ainda, "é vital que as IES preparem profissionais que sejam capazes de fazer o uso de seus conhecimentos, não apenas para si, mas também para auxiliar no entendimento das necessidades sociais e ambientais" (BECKER et al., 2015, p. 625).

Com isso, o objetivo deste estudo é analisar as inter-relações entre os constructos comportamentos e competências para a sustentabilidade em uma IES. Além disso, foram analisadas as diferenças entre os dois constructos com base no gênero, na faixa etária, no estado civil, na escolaridade, na renda, na categoria, no tempo de instituição e no exercício de cargo de chefia. O intuito maior é contribuir com a identificação de fatores que sejam promotores do desenvolvimento de uma Universidade Verde, ou seja, que sejam um ponto de partida para se refletir, criar e construir uma universidade mais sustentável por meio de seus servidores.

\section{Sustentabilidade: Comportamentos e Competências}

Com o objetivo de analisar o percurso da conscientização acerca da sustentabilidade, Elkington (1997) apresenta o desenvolvimento sustentável em 3 dimensões: sustentabilidade ecológica, social e econômica, e Foladori (2002) foca na parte social por ser tratar da dimensão que salienta a relevância da participação social e das potencialidades e qualidades dos indivíduos para se construir uma sociedade melhor e mais justa. Ele apresenta que a crise ambiental se estabeleceu nas décadas de 60 e 70, nas quais vários eventos passaram a discutir a necessidade de se repensar o desenvolvimento, visto que muitos danos estavam sendo causados à natureza devido ao interesse humano.

Alguns pré-requisitos fundamentais para que seja possível a prática da sustentabilidade são: democracia e estabilidade política, paz, respeito à lei e à propriedade, respeito aos instrumentos de mercado, ausência de corrupção, transparência e previsibilidade de governos, e reversão do atual quadro de concentração de renda nas esferas local e global (ALMEIDA, 2002). Não se trata, portanto, de uma tarefa simples e sim de um desafio que cabe não só às IES, mas a todas as organizações e a toda a sociedade. 
Os estudos relacionados à sustentabilidade provocam mudanças e elevam o conhecimento a respeito do tema, o que pode ser evidenciado na Figura 1, que apresenta as dimensões da sustentabilidade nos anos de 1993 e 2002 (SACHS, 1993, 2002).

Figura 1. Evolução das Dimensões da Sustentabilidade de Acordo com Sachs.

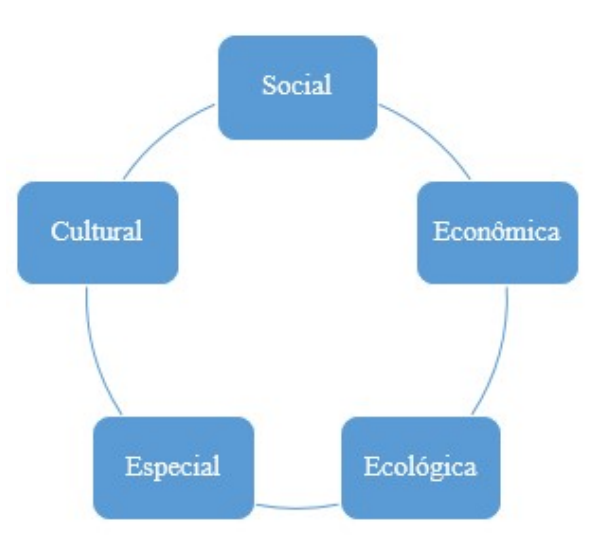

5 dimensões - 1993

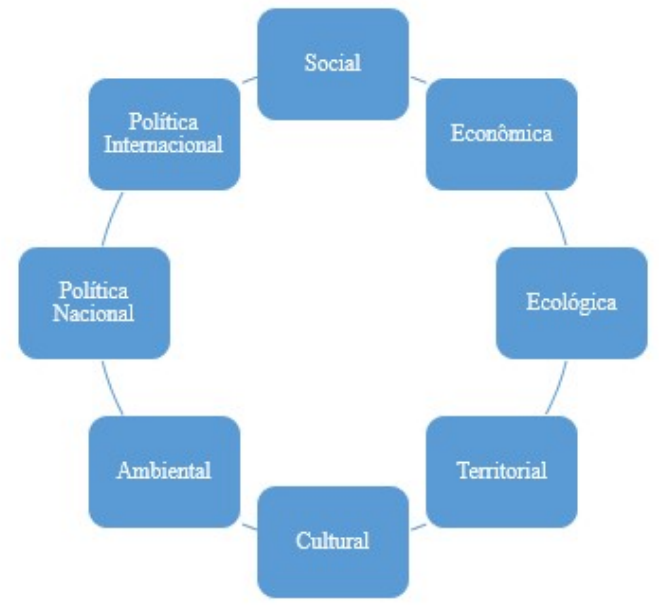

8 dimensões - 2002

Fonte: Elaborado pelos Autores.

O Quadro 1 apresenta características das oito dimensões da sustentabilidade, segundo Sachs (2002).

Quadro 1. Oito Dimensões da Sustentabilidade.

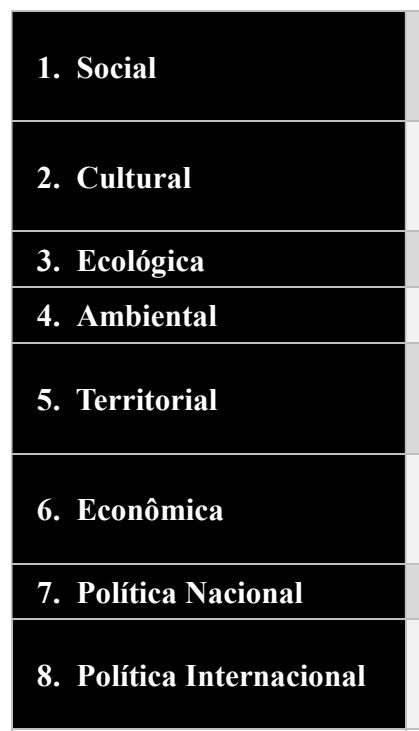

Equilíbrio e igualdade social, distribuição homogênea de renda, qualidade de vida.

Equilíbrio, tradição, inovação e autonomia em projetos nacionais e abertos para o mundo.

Preservação natural e limitação do uso dos recursos naturais.

Respeito aos ecossistemas naturais.

Equilíbrio na configuração rural-urbana, melhoria no ambiente urbano, desenvolvimento das regiões.

Equilíbrio econômico, segurança alimentar, modernização dos meios produtivos, desenvolvimento de pesquisas, inserção na economia internacional. Democracia, direitos humanos, projetos nacionais com empreendedores.

Promoção da paz, da cooperação mundial, do controle financeiro global, da gestão da diversidade natural e cultural e da cooperação científica e tecnológica.

Fonte: Sachs (2002).

Percebe-se, pelas dimensões consideras por Sachs (1993; 2002), que a sustentabilidade abrange muito mais do que questões ambientais. Sim, está preocupada com questões de ordem mundial, com as pesquisas tecnológicas que possam permitir melhorias na qualidade de vida das pessoas, na produção (seja rural ou industrial), com o respeito não só aos ecossistemas naturais, mas às culturas e às pessoas como um todo, e com a elaboração de políticas que proporcionem a união mundial em prol da sustentabilidade para todos.

Existem várias normas ambientais nacionais e internacionais relacionadas ao tema ambiental, como exemplo as normas britânicas BS 7750 (Especificações para Sistemas de Gestão Ambiental) e o padrão da União Europeia Eco-Managementand Audit Scheme (EMAS) que definiu critérios para certificações ambientais de processos 
industriais. No que se refere às normas brasileiras, tem-se as normas ISO (International Organization for Standardization) relativas às questões ambientais: ISO 14001 e ISO 14004. A ISO 14001 é conhecida como Sistemas de Gestão Ambiental - Especificações e Diretrizes para Uso, cujo objetivo é fornecer às organizações um sistema de gestão ambiental que permita o alcance dos objetivos ambientais e econômicos. Já a ISO 14004 é conhecida como Sistemas de Gestão Ambiental - Diretrizes gerais sobre princípios, sistemas e técnicas de apoio. Além dessas, tem-se as normas de auditoria ambiental ISO 19011 - Diretrizes para auditorias de sistema de gestão da qualidade e/ou ambiental (CAMPOS; LERÍPIO, 2009).

No que se refere à Gestão Pública, tem-se a Agenda 21: assinada em 1992, no Rio de Janeiro - Brasil, por 179 países na Conferência das Nações Unidas sobre o Meio Ambiente e o Desenvolvimento (CNUMAD) realizado pela Organização das Nações Unidas (ONU). O documento tem 40 capítulos que se constituem uma tentativa mais abrangente de promover o desenvolvimento sustentável, contemplando a proteção ao meio ambiente, a justiça social e a eficiência econômica. Percebe-se que, assim como a Política Nacional do Meio Ambiente, a Agenda 21 tem uma ampla abrangência que contempla as várias esferas da sociedade e que busca conciliar a vida em comunidade com a conservação e manutenção do meio ambiente.

Ainda, a Agenda Ambiental da Administração Pública (A3P), instituída em 1999 e fundamentada na Agenda 21, tem o objetivo de promover a Responsabilidade Socioambiental como política da administração pública, alinhando crescimento econômico com desenvolvimento sustentável. A A3P considera a importância que o Estado tem junto à sociedade no que se refere à Responsabilidade Socioambiental ao estimular iniciativas e ações que integrem sociedade, meio ambiente e interesse público.

Oliveira et al. (2013) consideram o conceito de sustentabilidade vinculada ao setor público como sustentabilidade pública que "pode ser entendida como a capacidade do ente público em subsistir ao tempo e aos governos (governabilidade) - princípio da continuidade do Estado (...)", de maneira a contemplar os aspectos ambientais, a legalidade, o orçamento público e as políticas econômico-sociais buscando garantir o bem comum ao povo (OLIVEIRA et al., 2013, p. 98). Ou seja, os autores julgam a relação entre sustentabilidade e serviço público por meio do princípio da continuidade, tendo, os dois, o objetivo comum de garantir o desenvolvimento e a manutenção das esferas que compõem o (meio) ambiente, assegurando de um lado - conceitualmente - a sobrevivência das gerações presentes e futuras (sustentabilidade) e, de outro lado, a prestação de serviços fundamentais (princípio da continuidade do serviço público).

Considerando que o poder público é responsável por assegurar qualidade de vida e garantir um meio ambiente equilibrado a todos, as ações de sua administração interna, em princípio, deveriam ser ambientalmente adequadas, com procedimentos visando, por exemplo, a redução de consumo de materiais e de serviços. Uma conduta de preservação do meio ambiente e de uso sustentável dos recursos naturais é esperada. As instituições públicas de meio ambiente teriam o dever de conhecer, a priori, os impactos socioambientais da degradação ambiental causada pela utilização abusiva dos recursos naturais e da grande geração de resíduos sólidos, líquidos e gasosos (CAIXETA, 2010, p. 1).

Felix (2007) aponta a urgência do trabalho educacional para a sensibilização na comunidade, para o pensamento sustentável, como forma de reverter e estabilizar os danos já causados ao meio ambiente, já que grande parte dos desequilíbrios, sejam eles ambientais ou sociais, se dá a partir do desperdício e do uso inadequado de bens da natureza. A partir das instituições de ensino, neste caso as universidades, pode-se alterar os hábitos e atitudes das pessoas, levando à formação de sujeitos mais conscientes.

A política ambiental deve estar ligada à missão, visão, valores e outras políticas da instituição. Percebe-se, assim, que a preocupação com a sustentabilidade perpassa o planejamento estratégico da organização, devendo estar alinhada aos objetivos e às atividades-fim (GOMES, 2010).

Ainda segundo Tauchen e Brandli (2006), é importante que as IES implantem um Sistema de Gestão Ambiental (SGA), já que podem ser pensadas enquanto núcleos urbanos devido a sua infraestrutura básica (redes de água, de energia, de saneamento, vias de acesso etc.). Os mesmos autores sugerem que:

Existem duas correntes de pensamento principais referentes ao papel das IES no tocante ao desenvolvimento sustentável. A primeira destaca a questão educacional como uma prática fundamental para que as IES, pela formação, possam contribuir na qualificação de seus egressos, futuros tomadores de decisão, para que incluam em 
suas práticas profissionais a preocupação com as questões ambientais. A segunda corrente destaca a postura de algumas IES na implementação de SGAs em seus campi universitários, como modelos e exemplos práticos de gestão sustentável para a sociedade (TAUCHEN e BRANDLI, 2006, p. 504).

Wang et al. (2013) ressaltam que a busca por uma sociedade ecologicamente correta requer muito apoio das instituições de ensino superior. Os autores fazem uma retomada quantitativa dos artigos publicados no Journal of Cleaner Production sobre os esforços acadêmicos para tratar da sustentabilidade no ensino superior, focando na educação, pesquisa, divulgação e execução de operações ecológicas no campus de diversas universidades no mundo. Porém, percebeu-se que os países desenvolvidos são os que mais apresentaram esses esforços acadêmicos, e países em desenvolvimento e emergentes são, portanto, desafiados a usufruir de seu conhecimento e experiências para contribuir com a construção de universidades verdes e com a educação para a sustentabilidade.

Tratar do tema sustentabilidade significa, na maior parte das vezes, tratar das atitudes e ações das pessoas, já que são elas as responsáveis pela manutenção do planeta, para melhor ou para pior. Considerando a influência das pessoas, indivíduos que constituem a sociedade e contribuem (ou não) para o desenvolvimento sustentável, este trabalho vai abordar as competências e comportamentos que as pessoas detêm em relação à sustentabilidade.

Comportamentos para a sustentabilidade abrangem atitudes e ações positivas em relação ao meio ambiente, significando consciência e intenção (ou não) que podem ser aprendidas, internalizadas e se transformar em rotina (PATO; TAMAYO, 2006). Pode ser, assim, considerado como "uma conduta efetiva, antecipada e dirigida à preservação do ambiente ou a minimização da degradação" (CORRAL-VERDUGO, 2006, p. 111).

O comportamento relacionado à sustentabilidade possui algumas variações na literatura, como por exemplo, comportamento verde ou comportamento pró ambiental, no qual se referem ao comportamento que possui um impacto positivo no ambiente (UNSWORTH; DMITRIEVA; ADRIASOLA, 2013). Ones e Dilchert (2013) afirmam que, sempre que comportamentos ambientais proativos são tomados no contexto do trabalho do indivíduo, eles se tornam comportamentos verdes empregados. Iqbal et al. (2018) atentam para a necessidade de sessões de treinamento extensivo e workshops, para que seja realizada uma ampla conscientização de todos os segmentos da vida, em cada funcionário.

Competências para a sustentabilidade são um conjunto de capacidades de desenvolvimento e/ou manutenção de processos participativos efetivos, estruturas de governança empoderadoras e promoção e incentivo de ciclos de feedback que institucionalizem mudanças (BRUNSTEIN; BOULOS, 2009). Dessa forma, competências para a sustentabilidade são ferramentas intrínsecas a cada indivíduo capazes de gerar atitudes e ações em prol da sustentabilidade.

Alguns estudos se propuseram a investigar as competências orientadas para a sustentabilidade em organizações (WILSON; LENSSEN; HIND, 2006; CARVALHO; STEFANO; MUNCK, 2015; CARVALHO; STEFANO; MUNCK, 2015; KUZMA; DOLIVEIRA; SILVA, 2017). Carvalho, Stefano e Munck (2015) identificaram a visão sistêmica, a tomada de decisão e a comunicação como competências fundamentais para a sustentabilidade. Ainda para os autores, as organizações diferenciam-se umas das outras com relação ao alinhamento destas competências com o desenvolvimento sustentável. Wilson, Lenssen e Hind (2006) identificaram três competências como importantes para o avanço da sustentabilidade nas organizações: competência para entender a interdependência entre empresas e a sociedade; competência para lidar com a diversidade; e competência de manter um diálogo significativo com os diferentes grupos de interesse da organização. Torna-se necessário salientar que os achados nos dois estudos possuem características semelhantes, salientando a importância da comunicação entre os atores. Por fim, Kuzma, Doliveira e Silva (2017) afirmam que o discurso existente entre a geração de competências organizacionais focadas em ações de sustentabilidade apresenta-se distante da realidade prática e nem sempre produz efeitos que impactam a dimensão operacional das organizações. Ainda, "a gestão pública deve desempenhar um papel relevante voltado para todos os segmentos da sociedade e buscar a reflexão e solução de soluções que permeiem a sustentabilidade" (GOES; MORALES, 2013, p. 202).

Os quadros 2 e 3 apresentam a composição dos constructos comportamentos e competências para a sustentabilidade. 
Quadro 2. Comportamentos para a Sustentabilidade.

\section{Comportamentos}

- Apago a luz quando saio de ambientes vazios;

- Evito ligar vários aparelhos elétricos ao mesmo tempo nos horários de maior consumo de energia;

- Evito desperdício de energia;

- Economizo água quando possível;

- Evito desperdício dos recursos naturais;

- Evito deixar o registro (torneira) aberta quando não preciso;

- Participo de programas e iniciativas que atuam na gestão de recursos hídricos e bacia hidrográfica;

- Evito jogar papel (lixo) no chão;

- Evito comprar produtos que são feitos de plástico;

- Separo o lixo conforme o tipo;

- Quando não encontro uma lixeira por perto guardo o resíduo para descartar em local adequado;

- Evito o desperdício de alimentos;

- Evito comer alimentos que contenham produtos químicos (conservantes ou agrotóxicos);

- Colaboro com a preservação da cidade onde vivo;

- Mobilizo as pessoas nos cuidados necessários para a conservação dos espaços públicos;

- Participo de atividades que cuidam do meio ambiente;

- Uso os transportes coletivos que tenho a minha disposição;

- Procuro andar a pé ou de bicicleta para ir para a universidade;

- Procuro compartilhar o veículo para usar o automóvel para mais de um ocupante.

Fonte: Elaborado por Beuron (2016) com base em (PATO; TAMAYO, 2006; MARINHO; GONÇALVES; KIPERSTOK, 2014); TRIVEDI, 2015; THOMASHOW, 2014; BARLA et al. 2015; KRIZEK et al., 2007; KAPLAN, 2015).

Quadro 3. Competências para a Ssustentabilidade.

\section{Competências}

- Tenho capacidade de fazer a gestão de conflitos;

- Consigo tomar decisões que levem ao Desenvolvimento Sustentável;

- Resolvo problemas relacionados ao Desenvolvimento Sustentável;

- Lido com incertezas;

- $\quad$ Penso de forma prospectiva;

- Aproveito as oportunidades econômicas para melhorar a subsistência e a qualidade de vida;

- Aplico os conhecimentos ao desenvolvimento sustentável;

- $\quad$ Penso de forma criativa e crítica;

- Crio e uso ferramentas;

- Reflito sobre os valores do desenvolvimento sustentável;

- Tenho boa comunicação oral e escrita;

- Divulgo informações relacionadas ao desenvolvimento sustentável.

Fonte: Elaborado pelos Autores com Base em UNESCO (2005), Barth et al. (2007), Wals (2014) e Gombert-Courvoisier et al. (2014).

Esses dois conjuntos - de comportamentos e competências para a sustentabilidade - são utilizados na presente pesquisa a fim de se evidenciar as inter-relações entre os dois constructos. 


\section{Método}

Este trabalho constitui-se por uma pesquisa quantitativa realizada por meio do método survey com aplicação de questionários com os servidores da instituição (GIL, 2009; HAIR et al., 2005). A pesquisa teve como base uma amostragem não-probabilística por conveniência dos servidores públicos federais da instituição (docentes e técnico-administrativos em educação - TAE's), que se dispuserem em responder ao questionário proposto a fim de contribuir com a pesquisa. Ao total são 4.729 servidores, sendo 1.943 docentes e 2.786 TAE's (dados do site da UFSM - UFSM em números, 2017) (BABBIE, 1999). O número amostral, calculado com 5\% de erro e $95 \%$ de confiança é 356 respondentes. A amostra constituiu-se do número de servidores que responderam ao questionário, num total de 549 respondentes.

Como instrumento base para a pesquisa, foi utilizado um questionário composto por três partes: a primeira parte refere-se ao perfil dos respondentes; a segunda parte refere-se aos comportamentos e a terceira parte referese às competências dos respondentes no que tange à sustentabilidade (comportamentos e competências para a sustentabilidade apresentados, respectivamente, nos quadros 2 e 3). São utilizadas duas escalas: uma que vai de um (nunca) a cinco (sempre) e outra que vai de um (nenhuma) a dez (muito). O questionário foi encaminhado por e-mail para o banco de dados que o Centro de Processamento de Dados da instituição. O instrumento utilizado teve como base o trabalho de Beuron (2016).

Os respondentes tiveram conhecimento quanto ao Termo de Consentimento Livre e Esclarecido e ao Termo de Confidencialidade. O projeto foi registrado na Plataforma Brasil e passou por aprovação do Comitê de Ética em Pesquisa (CEP) da instituição.

Foram realizados testes de normalidade a fim de verificar se os dados são paramétricos. Também foi feita a verificação de outliers para verificar se existe algum valor discrepante e se analisado, deve ser desconsiderado. Testes de correlação de Pearson foram efetuados para analisar as inter-relações entre os dois constructos. Os dados obtidos por meio dos questionários foram tratados no programa SPSS (Statistical Package for Social Sciences), versão 21. Foram analisadas as diferenças entre os dois constructos com base no gênero, na faixa etária, no estado civil, na escolaridade, na renda, na categoria, no tempo de instituição e no exercício de cargo de chefia.

Do total de 549 respondentes, 481 questionários foram considerados válidos e foram analisados neste estudo.

\section{Análise e Discurssão dos Resultados}

A relação entre os constructos comportamentos e competências foi evidenciada por meio da Correlação de Pearson e apresentada no Quadro 4.

Quadro 4. Correlação entre os Constructos.

\begin{tabular}{|c|c|c|c|}
\hline & & Comportamento & Competência \\
\hline \multirow{5}{*}{ 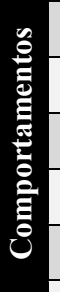 } & Correlação de Pearson & 1 & $0,486^{* *}$ \\
\hline & Sig. (2 extremidades) & & 0,000 \\
\hline & Soma dos quadrados e produtos cruzados & 280,981 & 126,591 \\
\hline & Covariância &, 585 & 0,264 \\
\hline & $\mathrm{N}$ & 481 & 481 \\
\hline \multirow{5}{*}{ 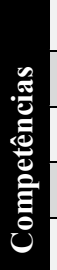 } & Correlação de Pearson & $0,486^{* *}$ & 1 \\
\hline & Sig. (2 extremidades) & 0,000 & \\
\hline & Soma dos quadrados e produtos cruzados & 126,591 & 241,331 \\
\hline & Covariância & 0,264 & 0,503 \\
\hline & $\mathrm{N}$ & 481 & 481 \\
\hline
\end{tabular}

**. A correlação é significativa no nível 0,01 (2 extremidades).

Fonte: Elaborado pelos Autores.

O quadro demonstra que há relação entre os construtos (Sig. < 0,01). Nesse caso, quando há comportamento tende a haver competência, e vice-versa. Esta relação é considerada moderada por Bisquerra, Sarriera e Martínez (2004), visto que o valor da relação ficou entre 0,4 e $0,6($ Pearson $=0,486)$. 
As competências (Quadro 3) resultaram em uma média de 3,87, considerando a escala likert de 1 a 5, e um desvio padrão variando em 0,709. Já os comportamentos (Quadro 2) tiveram uma média de 3,99 e um desvio padrão de 0,76. Cabe ressaltar que dois itens do construto comportamento foram excluídos desta e das demais análises pelo fato de não serem dados considerados normais.

\begin{tabular}{|c|c|c|}
\hline Item & Média & $\begin{array}{l}\text { Desvio } \\
\text { Padrão }\end{array}$ \\
\hline Comportamento & 3,99 & 0,76510 \\
\hline Competências & 3,87 & 0,70906 \\
\hline
\end{tabular}

Fonte: Dados da Pesquisa.

As médias dos construtos foram muito próximas, entretanto, os respondentes apresentam-se com maior comportamento do que competência.

Com o objetivo de analisar as relações entre os comportamentos e competências para a sustentabilidade, foi aplicada a técnica de Correlação de Pearson. O resultado é que existe correlação entre comportamento e competência. Há uma correlação positiva em 0,486 , ou seja, quando há um aumento na competência, verifica-se um aumento no comportamento e vice-versa. O servidor pode ter comportamento alto e competência alta, mas não se sabe ao certo qual constructo aparece primeiro. Pestana e Gageiro (2003) sugerem que as variações do coeficiente de Correlação entre 0,01 e 0,39 são classificadas como associações baixas; entre 0,4 e 0,69 são associações moderadas; entre 0,7 e 1 são consideradas associações altas. A correlação é moderada, de acordo com as faixas a seguir, significando que há mais chances de que um servidor com comportamento alto ter competência alta também, e vice-versa.

A seguir é feita a análise de acordo com as características dos participantes.

\subsection{Análise de Diferenças}

Esta seção tratará de apresentar testes buscando verificar diferenças de médias entre os construtos e a característica dos participantes do estudo.

Tabela 2. Análise de Médias dos Construtos e Gêneros.

\begin{tabular}{|c|c|c|c|c|c|c|}
\hline Construtos & Gênero & Média & $\begin{array}{c}\text { Desvio } \\
\text { Padrão }\end{array}$ & Levene & $t$ & Significância \\
\hline \multirow{2}{*}{ COMPORTAMENTO } & Feminino & 4,2944 & 0,67191 & \multirow{2}{*}{0,704} & \multirow{2}{*}{2,549} & \multirow{2}{*}{$0,011^{*}$} \\
\hline & Masculino & 4,1330 & 0,71582 & & & \\
\hline \multirow{2}{*}{ COMPETÊNCIA } & Feminino & 3,8831 & 0,68266 & \multirow{2}{*}{0,96} & \multirow{2}{*}{0,614} & \multirow{2}{*}{0,541} \\
\hline & Masculino & 3,8433 & 0,73705 & & & \\
\hline
\end{tabular}

* Significante ao nível de 0,05 .

Fonte: Dados da Pesquisa.

Existe diferença significativa no construto comportamento (Sig. $<0,05-$ teste $T$ ). Isto quer dizer que mulheres possuem comportamento mais pró-universidades verdes que homens.

Tabela 3. Análise de Médias dos Construtos e Faixa Etária.

\begin{tabular}{|c|c|c|c|c|c|c|}
\hline Construtos & Faixa Etária & Média & $\begin{array}{l}\text { Desvio } \\
\text { Padrão }\end{array}$ & Levene & $z$ & Significância \\
\hline \multirow{4}{*}{ COMPORTAMENTO } & Até 29 anos & 4,3167 & 0,67627 & \multirow{4}{*}{1,300} & \multirow{4}{*}{3,7724} & \multirow{4}{*}{$0,001 *$} \\
\hline & De 30 a 35 anos & 4,4565 & 0,60060 & & & \\
\hline & De 36 a 41 anos & 4,2174 & 0,67655 & & & \\
\hline & De 42 a 47 anos & 4,1207 & 0,70282 & & & \\
\hline
\end{tabular}




\begin{tabular}{|c|c|c|c|c|c|c|}
\hline & De 48 a 53 anos & 4,2034 & 0,73765 & & & \\
\hline & De 54 a 59 anos & 4,0225 & 0,69048 & & & \\
\hline & 60 anos ou mais & 4,0645 & 0,81386 & & & \\
\hline \multirow{7}{*}{ COMPETÊNCIA } & Até 29 anos & 3,8917 & 0,65154 & \multirow{7}{*}{2,049} & \multirow{7}{*}{3,777} & \multirow{7}{*}{$0,001^{*}$} \\
\hline & De 30 a 35 anos & 4,0489 & 0,69163 & & & \\
\hline & De 36 a 41 anos & 4,0272 & 0,66450 & & & \\
\hline & De 42 a 47 anos & 3,7414 & 0,68978 & & & \\
\hline & De 48 a 53 anos & 3,7458 & 0,70327 & & & \\
\hline & De 54 a 59 anos & 3,7360 & 0,72335 & & & \\
\hline & 60 anos ou mais & 3,5968 & 0,81055 & & & \\
\hline
\end{tabular}

*significante ao nível de 0,05 .

Fonte: Dados da Pesquisa.

Ambos os construtos apresentaram diferença significativa entre as médias (Sig. $<0,05-$ Teste Anova). Pessoas de menor idade possuem mais comportamento e competências.

Tabela 4. Análise de Médias dos Construtos e Estado Civil.

\begin{tabular}{|c|c|c|c|c|c|c|}
\hline Construtos & Estado Civil & Média & $\begin{array}{l}\text { Desvio } \\
\text { Padrão }\end{array}$ & Levene & $z$ & Significância \\
\hline \multirow{4}{*}{ COMPORTAMENTO } & Solteiro & 4,1702 & 0,68616 & \multirow{4}{*}{0,891} & \multirow{4}{*}{1.042} & \multirow{4}{*}{0,374} \\
\hline & $\begin{array}{l}\text { Casado ou união } \\
\text { estável }\end{array}$ & 4,2143 & 0,70469 & & & \\
\hline & Divorciado & 4,3947 & 0,63839 & & & \\
\hline & Outro & 4,2500 & 0,88641 & & & \\
\hline \multirow{4}{*}{ COMPETÊNCIA } & Solteiro & 3,7730 & 0,68263 & \multirow{4}{*}{0,885} & \multirow{4}{*}{1,711} & \multirow{4}{*}{0,164} \\
\hline & $\begin{array}{l}\text { Casado ou união } \\
\text { estável }\end{array}$ & 3,8810 & 0,71511 & & & \\
\hline & Divorciado & 4,0395 & 0,69147 & & & \\
\hline & Outro & 4,0000 & 0,92582 & & & \\
\hline
\end{tabular}

Fonte: Dados da Pesquisa.

Não observou-se diferença de média entre os estados civis e os construtos (Sig. > 0,05 - Teste Anova). Pessoas de diversos estados civis possuem o mesmo comportamento e competências.

Tabela 5. Análise de Médias dos Construtos e Escolaridade.

\begin{tabular}{|c|c|c|c|c|c|c|}
\hline Construtos & Escolaridade & Média & $\begin{array}{l}\text { Desvio } \\
\text { Padrão }\end{array}$ & Levene & $z$ & Significância \\
\hline \multirow{7}{*}{ COMPORTAMENTO } & Ensino Fundamental & 5,0000 & 0,00000 & \multirow{7}{*}{3,094} & \multirow{7}{*}{2,117} & \multirow{7}{*}{$0,049^{*}$} \\
\hline & Ensino Médio & 4,4722 & 0,69636 & & & \\
\hline & Ensino Superior & 4,2195 & 0,75399 & & & \\
\hline & Especialização & 4,1901 & 0,69898 & & & \\
\hline & Mestrado & 4,1171 & 0,67067 & & & \\
\hline & Doutorado & 4,2143 & 0,70711 & & & \\
\hline & Pós-doutorado & 4,2759 & 0,52757 & & & \\
\hline
\end{tabular}




\begin{tabular}{|c|c|c|c|c|c|c|}
\hline \multirow{7}{*}{ COMPETÊNCIA } & Ensino Fundamental & 4,7500 & 0,50000 & \multirow{7}{*}{1,267} & \multirow{7}{*}{2,629} & \multirow{7}{*}{$0,017^{*}$} \\
\hline & Ensino Médio & 4,1250 & 0,62536 & & & \\
\hline & Ensino Superior & 3,8476 & 0,73572 & & & \\
\hline & Especialização & 3,8595 & 0,72233 & & & \\
\hline & Mestrado & 3,7342 & 0,72557 & & & \\
\hline & Doutorado & 3,9082 & 0,63953 & & & \\
\hline & Pós-doutorado & 3,8276 & 0,73528 & & & \\
\hline
\end{tabular}

*significante ao nível de 0,05 .

Fonte: Dados da Pesquisa.

Ambos os construtos apresentaram diferença significativa entre as médias (Sig. $<0,05-$ Teste Anova). Pessoas com o nível mais baixo de escolaridade apresentam maiores índices de comportamento e competência.

Tabela 6. Análise de Médias dos Construtos e Renda.

\begin{tabular}{|c|c|c|c|c|c|c|}
\hline Construtos & Renda & Média & Desvio Padrão & Levene & $z$ & Significância \\
\hline \multirow{3}{*}{ COMPORTAMENTO } & Classe C & 4,2530 & 0,70442 & \multirow{3}{*}{0,887} & \multirow{3}{*}{0,712} & \multirow{3}{*}{0545} \\
\hline & Classe B & 4,1404 & 0,68971 & & & \\
\hline & Classe A & 4,2087 & 0,69454 & & & \\
\hline \multirow{3}{*}{ COMPETÊNCIA } & Classe C & 3,8775 & 0,72544 & \multirow{3}{*}{0,812} & \multirow{3}{*}{0,278} & \multirow{3}{*}{0,241} \\
\hline & Classe B & 3,8202 & 0,67878 & & & \\
\hline & Classe A & 3,8826 & 0,69878 & & & \\
\hline
\end{tabular}

Fonte: Dados da Pesquisa.

Aqui é interessante ressaltar que nenhum dos respondentes, na Tabela 6, demarcou a classe E, e apenas quatro marcaram classe $\mathrm{C}$, estes não foram computados por ser uma amostra muito pequena. Porém, ambos os construtos não apresentaram diferença significativa entre as médias (Sig. $<0,05-$ Teste Anova). Pessoas de diferentes rendas apresentam o mesmo nível de comportamento e competência.

Tabela 7. Análise de Médias dos Construtos e Categoria.

\begin{tabular}{c|l|l|l|l|l||c|c|}
\hline \multicolumn{1}{c|}{ Construtos } & Categoria & Média & \multicolumn{1}{|c|}{ Desvio Padrão } & Levene & \multicolumn{2}{c|}{ Sig. } \\
\hline \multirow{2}{*}{ COMPORTAMENTO } & Docente & 4,2348 & 0,66378 & \multirow{2}{*}{0,278} & \multirow{2}{*}{0,371} & \multirow{2}{*}{0,711} \\
\cline { 2 - 5 } & Técnico & 4,2092 & 0,71061 & & & \\
\hline \multirow{2}{*}{ COMPETÊNCIA } & Docente & 3,8788 & 0,65125 & \multirow{2}{*}{0,022} & \multirow{2}{*}{0,299} & 0,765 \\
\cline { 2 - 5 } & Técnico & 3,8582 & 0,73055 & & & \\
\hline
\end{tabular}

Fonte: Dados da Pesquisa.

Percebe-se que não existem diferença significativa nos construtos pesquisados (Sig. $>0,05-$ teste $T$ ). Isto quer dizer que tanto docentes como técnicos possuem o mesmo nível de comportamento e competência. A homocedasticidade foi feita pelo teste de Levene, e o resultado indica haver igualdade de variância apenas para o construto comportamento, visto que o resultado ficou acima de 0,05 (HAIR JR et al., 2005). O construto competência não apresentou variâncias iguais.

Tabela 8. Análise de Médias dos Construtos e Tempo de Instituição.

\begin{tabular}{|c|c|c|c|c|c|c|}
\hline Construtos & Tempo de Instituição & Média & $\begin{array}{l}\text { Desvio } \\
\text { Padrão }\end{array}$ & Levene & $z$ & Sig. \\
\hline \multirow{2}{*}{ COMPORTAMENTO } & Menos de 1 ano & 3,8235 & 0,62622 & \multirow{2}{*}{0,680} & \multirow{2}{*}{7,114} & \multirow{2}{*}{$0,000^{*}$} \\
\hline & De 1 a menos de 3 anos & 4,0968 & 0,71768 & & & \\
\hline
\end{tabular}




\begin{tabular}{|c|c|c|c|c|c|c|}
\hline & De 3 a menos de 5 anos & 4,1029 & 0,71529 & & & \\
\hline & De 5 a menos de 10 anos & 4,1574 & 0,69929 & & & \\
\hline & Mais de 10 anos & 4,3828 & 0,65552 & & & \\
\hline \multirow{5}{*}{ COMPETÊNCIA } & Menos de 1 ano & 3,6618 & 0,65967 & \multirow{5}{*}{1,329} & \multirow{5}{*}{4,174} & \multirow{5}{*}{$0,002 *$} \\
\hline & De 1 a menos de 3 anos & 3,8629 & 0,62180 & & & \\
\hline & De 3 a menos de 5 anos & 3,6912 & 0,70197 & & & \\
\hline & De 5 a menos de 10 anos & 3,7731 & 0,71494 & & & \\
\hline & Mais de 10 anos & 4,0000 & 0,71891 & & & \\
\hline
\end{tabular}

* significante ao nível de 0,05

Fonte: Dados da Pesquisa.

Ambos os construtos apresentaram diferença significativa entre as médias (Sig. $<0,05-$ Teste Anova). Servidores que estão há mais tempo possuem maior nível de comportamento e competências.

Tabela 9. Análise de Médias dos Construtos e Cargo de Chefia.

\begin{tabular}{|c|c|c|c|c|c|c|}
\hline Construtos & $\begin{array}{c}\text { Cargo de } \\
\text { Chefia }\end{array}$ & Média & Desvio Padrão & Levene & $t$ & Sig. \\
\hline \multirow{2}{*}{ COMPORTAMENTO } & Sim & 4,2308 & 0,69926 & \multirow{2}{*}{0,854} & \multirow{2}{*}{0,278} & \multirow{2}{*}{0,781} \\
\hline & Não & 4,2108 & 0,69775 & & & \\
\hline \multirow{2}{*}{ COMPETÊNCIA } & Sim & 3,8308 & 0,68640 & \multirow{2}{*}{0,557} & \multirow{2}{*}{0,635} & \multirow{2}{*}{0,534} \\
\hline & Não & 3,8761 & 0,71785 & & & \\
\hline
\end{tabular}

Fonte: Dados da Pesquisa.

Percebe-se que não existem diferença significativa nos construtos pesquisados ( $\mathrm{Sig} .>0,05-$ teste T). Isto quer dizer que tanto as pessoas que possuem cargo de chefia como as que não possuem têm o mesmo nível de comportamento e competência.

Em todos os casos (com exceção da Categoria), a homocedasticidade foi feita pelo teste de Levene, e o resultado indica haver igualdade de variância, um requisito necessário para o teste de Anova (HAIR JR et al., 2005).

De maneira geral, a pesquisa identificou que existe uma correlação positiva entre comportamento e competência (quando aumenta a competência, aumenta o comportamento e vice-versa). Foi verificado que o servidor pode ter comportamento alto e competência alta, mas não se sabe ao certo qual constructo aparece primeiro, ou qual influência ou é influenciado pelo outro. Além disso, os respondentes apresentam maiores índices de comportamento (Quadro 2) do que de competência (Quadro 3).

O estudo também mostra que mulheres possuem comportamento mais pró-universidades verdes; pessoas de menos idade possuem mais comportamento e competências; pessoas com o mais baixo nível de escolaridade apresentam maiores índices de comportamento e competência e pessoas de diferentes rendas apresentam o mesmo nível de comportamento e competência.

Também foi identificado que tanto docentes quanto técnicos possuem o mesmo nível de comportamento e competência e que servidores que estão há mais tempo possuem maior nível de comportamento e competências.

\section{Considerações Finais}

Este estudo buscou analisar as inter-relações entre os constructos comportamentos e competências apresentados nos Quadros 2 e 3, respectivamente - para a sustentabilidade dos servidores de uma instituição de ensino superior.

Verificou-se que existe uma correlação positiva entre comportamento e competência (quando aumenta a competência, aumenta o comportamento e vice-versa). O servidor pode ter comportamento alto e competência alta, mas não se sabe ao certo qual constructo aparece primeiro. Os respondentes apresentam maiores índices de comportamento do que de competência. 
Segundo o estudo, mulheres possuem comportamento mais pró-universidades verdes que homens; pessoas de menos idade possuem mais comportamento e competências; não observou-se diferença de média entre os estados civis e os construtos (pessoas de diversos estados civis possuem o mesmo comportamento e competências); pessoas com o mais baixo nível de escolaridade apresentam maiores índices de comportamento e competência; pessoas de diferentes rendas apresentam o mesmo nível de comportamento e competência.

No que se refere à categoria, tanto docentes quanto técnicos possuem o mesmo nível de comportamento e competência. Servidores que estão há mais tempo possuem maior nível de comportamento e competências. Servidores que possuem cargo de chefia e que não possuem têm o mesmo nível de comportamento e competência.

No que se refere ao papel das universidades para o desenvolvimento sustentável, elas devem ser lugares de investigação acadêmica, de aprendizagem e de liderança, sendo capaz de tratar de assuntos como inclusão social, valorizar sistemas éticos, integrar a sustentabilidade no ensino, estudar e promover melhores práticas e ferramentas para o desenvolvimento sustentável: " a visão da educação para o desenvolvimento sustentável é a de um mundo onde todos tenham a oportunidade de se beneficiar de uma educação de qualidade e de aprender os valores, comportamento e estilos de vida requeridos para o desenvolvimento sustentável" (UNESCO, 2005, p. 65).

Importante salientar que "desenvolvimento sustentável é um conceito dinâmico que reconhece que a sociedade humana está em constante transformação" (UNESCO, 2005, p. 39). Deve-se prezar pelo respeito aos direitos humanos, pelo cuidado com a comunidade que convive em meio à diversidade cultural, pela proteção e preservação do meio ambiente e pela cultura da não-violência, da tolerância e da paz. Para isso, "a educação deve inspirar a crença que cada um de nós tem o poder e a responsabilidade de introduzir mudanças positivas em escala global" (UNESCO, 2005, p. 43).

\section{Agradecimentos}

Agradecimento ao Conselho Nacional de Desenvolvimento Científico e Tecnológico (CNPq) e à Universidade Federal de Santa Maria (UFSM).

\section{Referências}

ALMEIDA, F. O bom negócio da sustentabilidade. Editora Nova Fronteira, Rio de Janeiro, 2002.

BARLA, P. et al. Reducing automobile dependency on campus using transport demand management: a case study for Quebec City. Canadian Public Policy, Analyse de politiques, march. 2015.

BARTH, M. et al. Developing key competencies for sustainable development in higher education. International Journal of Sustainability in Higher Education, v. 8, n. 4, p. 416-430, 2007.

BISQUERRA, R.; et al. Introdução à Estatística: enfoque informático com o pacote estatístico SPSS. Porto Alegre: Artmed, 2004.

BRUNSTEIN, J.; BOULOS, S. A dimensão política da competência dos executivos em sua relação com stakeholders. In: XXXIII Encontro Nacional da AnPAD. Anais, São Paulo, 2009. Disponível em: .< http://www.anpad.org.br/admin/pdf/EOR2123.pdf $>$.

CAIXETA, D. M. Atitudes e comportamentos ambientais: um estudo comparativo entre servidores de instituições públicas federais. Dissertação de Mestrado. Brasília, 2010.

CAMPOS, L. M. S; LERÍPIO, A. A. Auditoria Ambiental: uma ferramenta de gestão. São Paulo: Atlas, 2009.

CARVALHO, A. C. V.; STEFANO, S. R.; MUNCK, L. Competências voltadas à sustentabilidade organizacional: um estudo de caso em uma indústria exportadora. Gestão\& Regionalidade, v. 3, n. 1, p. 33-48, 2015.

CMMAD - Comisão Mundial sobre Meio Ambiente Desenvolvimento. Nosso futuro comum. Rio de Janeiro: FGV, 1991.

CORRAL-VERDUGO, V. Contribuciones del análisis de la conducta a la investigación del comportamiento proecológico. Revista Mexicana de Análisis de laConducta, México, v.32, n. 002, p. 111-128, 2006. 
DECKER, D. V., et al. Educação para a sustentabilidade no Ensino Superior: O papel do docente na formação do Administrador. Revista Eletrônica em Gestão, Educação e Tecnologia Ambiental, Santa Maria, v. 19, n. 3 , p. 615-628, 2015.

ELKINGTON, J. Cannibals with forks: the triple bottom line of 21 st century susiness. Gabriola Island: Chapstone Publishing, 1997

FELIX, R. A. Z. Coleta Seletiva em Ambiente Escolar. Revista Eletrônica do Mestrado em Educação Ambiental, Rio Grande do Sul, v. 18, p. 56-71. 2007.

FOLADORI, G. Avanços e limites da sustentabilidade social. Revista Paranaense de Desenvolvimento. Curitiba, no 102 , p. 103-113, 2002.

GAZZONI, F., et al. O papel das IES no desenvolvimento sustentável: estudo de caso da Universidade Federal de Santa Maria. Revista Gestão Universitária na América Latina - GUAL. Florianópolis, v. 11, n. 1, p. 48$70,2018$.

GOES, G. A., MORALES, A. G. Gestão pública e sustentabilidade: desafios, ações e possibilidades. In.: IX Fórum Ambiental da Alta Paulista, v. 9, n. 4, p. 199-212, 2013.

GOMBERT-COURVOISIER, S. et al. Higher Education for Sustainable Consumption: case report on the Human Ecology Master' s course (University of Bordeaux, France), Journal of Cleaner Production, n. 62, p. 8288, 2014. Disponível em: <http://dx.doi.org/10.1016/j.jclepro.2013.05.032>. Acesso em 01/06/2016.

GOMES, L. P. A gestão de resíduos na Universidade do Vale do Rio dos Sinos (Unisinos) atendendo aos requisitos da ISO 14001:2004. In: CONTO, S. M. Gestão de Resíduos em Universidades. Caxias do Sul: Educs, 2010. Cap. 3, p. 61-86.

HAIR, J. et al. Análise Multivariada de Dados. 5ª Ed. Porto Alegre: Bookman, 2005.

IQBAL, Q.; et al. Employee's green behavior for environmental sustainability: a case of banking sector in Pakistan, World Journal of Science, Technology and Sustainable Development, v. 15, n. 2, p.118-130, 2018.

KAPLAN, D. H. Transportation sustainability on a university campus, International Journal of Sustainability in Higher Education, v. 16, Iss 2, pp. 173-186, 2015. Disponível em: <http://dx.doi.org/10.1108/IJSHE-032013-0023>. Acesso em 15 abr.2015.

KRIZEK, K.J. et al. Analysing the benefis and costs of bicycle facilities via online guidelines, Planning, Practice \& Research, v. 22, n. 2, p.197-213, 2007.

KUZMA, E. L.; DOLIVEIRA, S. L. D.; SILVA, A. Q. Competências para a sustentabilidade organizacional: uma revisão sistemática, Caderno EBAPE.BR, v. 15, Edição Especial, p. 428-444, 2017.

MARINHO, M. et al. Water conservation as a tool to support sustainable practices in a Brazilian public university. Journal of Cleaner Production, n. 62 p. 98-106, 2014. Disponível em: $<$ http://dx.doi.org/10.1016/j.jclepro.2013.06.053>. Acesso em: 15 abr.2015.

MELETI, M. V., FADEL, B., SMITH, M. Perspectiva sistêmica da sustentabilidade nas organizações sob a ótica da cultura organizacional e sua repercussão no desenvolvimento regional. $8^{\circ}$ Congresso Brasileiro de Sistemas. Revista Gestão \& Conhecimento. ISSN 1808-6594. Minas Gerais, 2012. Disponível em: $<$ http://www.pucpcaldas.br/graduacao/administracao/revista/artigos/esp1_8cbs/artigos_8cbs_2012.html> 
Acesso em: 17/01/2018.

MINISTÉRIO DO MEIO AMBIENTE. Agenda 21 Global. Disponível em: $<\underline{\text { http://www.mma.gov.br/responsabilidade-socioambiental/agenda-21/agenda-21-global }>}$ Acesso em $22 / 07 / 2016$.

, Agenda Ambiental na Administração Pública - A3P. Disponível em: < http://www.mma.gov.br/destaques/item/8852> Acesso em: 22/07/2016.

OLIVEIRA, A. G., CARVAlHO, H. A., CORRÊA, D. P., Governança Pública e governabilidade: Accountability e Disclosure possibilitadas pela Contabilidade Aplicada ao Setor Público como Instrumento de Sustentabilidade do Estado. Revista de Educação e Pesquisa em Contabilidade. Repec, Brasília, v. 7, n. 1, art. 6, p. 91-104, 2013.

ONES, D. S.; DILCHERT, S. Measuring, understanding, and influencing employee green behaviors In HUFFMAN, A. H.; KLEIN, S. R. Green organizations: Driving change with I-O psychology, Routledge, 2013.

PATO, C. M. L., TAMAYO, A. A Escala de Comportamento Ecológico: desenvolvimento e validação de um instrumento de medida. Estudos de Psicologia, 11(3), 289-296, 2006.

PESTANA, M. H., GAGEIRO, J. N. Análise de dados para ciências sociais: a complementariedade do SPSS. Lisboa: Silabo, 2003.

RODRIGUES, M. S. B. Crenças ambientais e comportamentos ecológicos de usuários do restaurante universitário da Universidade de Brasília. Dissertação de mestrado. Brasília, 2011.

SACHS, I. Estratégias de transição para o século XXI: desenvolvimento e meio Ambiente. São Paulo: Studio Nobel e Fundação de Desenvolvimento Administrativo (FUNDAP), 1993.

, Caminhos para o desenvolvimento sustentável. Rio de Janeiro: Garamond, 2002.

SEBAstião, I. L. C., Aplicação da Pegada Ecológica ao Turismo. Como a Pegada Ecológica pode Influenciar a Gestão Ambiental. Universidade Nova de Lisboa. Lisboa, 2010. Disponível em: < https://run.unl.pt/bitstream/10362/4988/1/Sebastiao_2010.pdf > Acesso em: 10/01/2018.

SILVA, S. S., REIS, R. P., AMÂNCIO, R. Conceitos atribuídos à sustentabilidade em organizações de diferentes setores. Revista de Ciências da Administração. V. 16, n. 40, p. 90-103, 2014. Disponível em: $<$ http://dx.doi.org/10.5007/2175-8077.2014v16n40p90>. Acesso em: 06/06/2016.

TAUCHEN, J. A., BRANDLI, L. L. A gestão ambiental em instituições de ensino superior: modelo para implantação em campus universitário. Gestão \& Produção. V. 13, p. 503-515, set-dez, 2006.

TREVISAN, M. A ecologia industrial e as teorias de sistemas, institucional e da dependência de recursos a partir dos atores de um parque tecnológico. Tese de Doutorado. Universidade Federal do Rio Grande do Sul. Porto Alegre - $\quad$ RS, $2013 . \quad$ Disponível em: $<$ http://www.lume.ufrgs.br/bitstream/handle/10183/78035/000898433.pdf;sequence=1 $>$ Acesso em: $<$ $23 / 12 / 2016$.

THOMASHOW, M. The nine elements of sustainable campus. USA: Massachusetts Institute of Technology, 2014. 
TRIVEDI, B.G. Food Waste Prevention and Management in Higher Education. In: LEAL FILHO, W. et al. Implementing campus greening initiatives: approaches, methods and perspectives. World Sustainability Series. London: Springer, 2015.

UNESCO - United Nations Educational, Scientific and Cultural Organization. United Nations Decade of Education for Sustainable Development 2005-2014: Draft International Implementation Scheme, 2005. Disponível em: < http://unesdoc.unesco.org/images/0013/001399/139937e.pdf> Acesso em: 28/05/2017.

Década das Nações Unidas da Educação para o Desenvolvimento Sustentável 2005-2014. Documento Final Plano Internacional de Implementação. Brasília, 2005.

UNSWORTH, K. L.; DMITRIEVA, A.; ADRIASOLA, E. Changing behaviour: Increasing the effectiveness of workplace interventions in creating pro-environmental behaviour change, Journal of Organizational Behaviour, v. 34, n. 2, p. 211-229, 2013.

WALS, A. E. J. Sustainability in higher education in the context of the UM DESD: a review of learning and institutionalization processes, Journal of Cleaner Production, n. 62, p. 8-15, 2014. Disponível em: $<$ http://dx.doi.org/10.1016/j.jclepro.2013.06.007>. Acesso em 01/06/2016.

WANG, Y, et al. Moving towards an ecologically sound society? Starting from green universities and environmental higher education. Journal of Cleaner Production 61, 1-5, 2013. Disponível em: $<$ http://www.sciencedirect.com/science/article/pii/S0959652613006501>Acessoem: 13/12/2016.

WILSON, A.; LENSSEN, G.; HIND, P. Leadership qualities and management competencies for corporate responsibility: a research report for the European business in society. European Academy of Business in

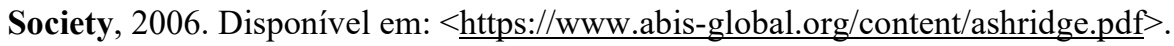

